\title{
Psychometric properties of the Brazilian Intolerance of Uncertainty Scale - Short Version (IUS-12)
}

\author{
Roberta P. Kretzmann, ${ }^{1}$ (D) Gustavo Gauer $^{1} \mathbb{D}$
}

\begin{abstract}
Introduction: The Intolerance of Uncertainty Scale - Short Version (IUS-12) is a measure of trait intolerance of uncertainty.

Objective: The purpose of the present study was to conduct a cross-cultural adaptation of the IUS-12 for use in Brazil and to investigate the scale's psychometric properties.

Methods: The research was conducted via an online research platform with a sample $(n=704 ; 80.1 \%$ female and $19.9 \%$ male) from different states in all five regions of Brazil. Participants were adults between 18 and 59 years of age $($ mean $=26.74$; standard deviation $=8.36$ ) who completed the Brazilian version of the IUS-12 online along with other anxiety-related measures.

Results: Confirmatory factor analysis demonstrated that the original two-dimensional structure fit the sample well. The total score for the scale had good internal consistency (Cronbach's alpha $[a]=0.88$ ), as did both subscales (i.e. Prospective IU $a=0.79$; Inhibitory IU $a=0.86$ ).

Conclusions: The results demonstrated strong positive correlations with measures of anxiety-related constructs, contributing to the transdiagnostic understanding of IU. The IUS-12 appears to be a useful tool for assessment of IU and its availability has several implications of theoretical importance and practical utility for understanding of psychopathology and uncertainty.

Keywords: Intolerance of uncertainty, psychometrics, social phobia, worry, anxiety disorders.
\end{abstract}

\section{Introduction}

As the world grows more complex, uncertainty, which can be considered "lack or incompleteness of information", has become pervasive in everyday situations. ${ }^{1}$ Decisionmaking under uncertainty encompasses the inability to predict future events accurately along with an absence of knowledge concerning the probabilities associated with the possible consequences. ${ }^{2,3}$ Therefore, uncertainty is perceived as an experience of the unknown with respect to the implications of events and of decisions made. ${ }^{4}$
Theoretical models have considered uncertainty a central feature in the understanding of psychopathology, especially in anxiety-related disorders. ${ }^{5}$ One of the key components of anxiety is the interpretation of environmental stimuli as threatening, which is associated with a negative evaluation of possible future consequences. The inability to tolerate uncertainty related to potential threat is therefore a central cognitive feature in the anxiety experience. ${ }^{4}$

Initially, intolerance of uncertainty (IU) was only associated with generalized anxiety disorder (GAD)

\footnotetext{
${ }^{1}$ Universidade Federal do Rio Grande do Sul (UFRGS), Porto Alegre, RS, Brazil.

This manuscript is part of the first author's Master's dissertation, presented on April 16, 2018, at Programa de Pós-Graduação em Psicologia, Universidade Federal do Rio Grande do Sul (UFRGS), Porto Alegre, RS, Brazil.

Submitted Nov 20 2018, accepted for publication Sep 012019.

Suggested citation: Kretzmann RP, Gauer G. Psychometric properties of the Brazilian Intolerance of Uncertainty Scale - Short Version (IUS-12). Trends Psychiatry Psychother. 2020;42(2):129-137. http://dx.doi.org/10.1590/2237-6089-2018-0087
} 
and excessive worry and was considered a "relatively broad construct representing cognitive, emotional and behavioral reactions to uncertainty in everyday life situations". ${ }^{6}$ The definition of IU has evolved since the 1990s, in line with extensive research on the subject. No univocal conceptual definition emerged over the course of these years and concepts evolved considerably as researchers turned their attention to the construct and its implications in clinical practice. ${ }^{7}$ This process is illustrated by the creation, and later adaptation, of a range of scales designed to assess IU.

Study of correlations between IU and other main constructs in anxiety-related disorders has led to emergence of a new definition, linked to the concept of fundamental fears. Current available evidence suggests that the construct may reflect a "dispositional characteristic that reflects a set of negative beliefs about uncertainty and its implications". ${ }^{7}$ Intolerance of uncertainty also represents an underlying fear of the unknown, ${ }^{7}$ which is triggered by a perceived lack of information that creates a propensity to be afraid. ${ }^{8}$ Accordingly, IU and fear of the unknown have been considered fundamental cognitive processes underlying all anxiety disorders. ${ }^{9}$

The Intolerance of Uncertainty Scale - Short Version (IUS-12) has 12 items with the same five-point Likert scale response format as the original scale (IUS-27). ${ }^{6}$ The IUS-12 is composed of two subscales: Prospective IU, which relates to a desire for predictability, and Inhibitory IU, which relates to paralysis caused by uncertainty. ${ }^{7}$ The Prospective IU subscale refers to cognitive assessments related to future uncertainties (e.g., "one should always look ahead so as to avoid surprises"), while the Inhibitory IU subscale covers behavioral manifestations of inhibition related to uncertainty (e.g. "when it's time to act, uncertainty paralyses me"). ${ }^{10}$

Several studies have demonstrated the stability of a two-factor structure for the IUS-12 and associations between these factors and different anxiety disorders. ${ }^{11-13}$ For example, the Prospective IU subscale seems to be more related to GAD and obsessive-compulsive disorder (OCD) symptoms, while Inhibitory IU is more associated with symptoms of social phobia, panic disorder, post-traumatic stress disorder (PTSD), and depression. ${ }^{10,11,14,15}$

Evidence of convergent validity, discriminant validity, and factor stability has been established. ${ }^{10}$ The IUS12 exhibits a strong correlation with the IUS-27 ( $r=$ 0.96 ) and subsequent analyses with IUS-12 using large samples demonstrated the reliability of the two-factor structure. ${ }^{10,16}$ The overall scale has excellent general internal consistency (Cronbach's alpha [a] $=0.92$ ), as do the Prospective IU ( $a=0.87$ ) and Inhibitory IU ( $a=$ 0.90 ) subscales. $^{17}$

The IUS-12 is based on a definition of general uncertainty-related reactions that may be present in different anxiety disorders. The scale is considered a measure of intolerance of uncertainty comparable to the original scale. ${ }^{13,18}$ Accordingly, the IUS-12 has shown suitable psychometric properties and has been considered a useful tool in the transdiagnostic evaluation of trait IU. ${ }^{5}$

Current extensive evidence suggests positive correlations between IU and anxiety-related constructs such as worry, ${ }^{7}$ anxiety sensitivity, ${ }^{17}$ obsessions and compulsions, ${ }^{12}$ and fear of negative evaluation. ${ }^{19}$ With regard to anxiety-related disorders per se, IU has been related to social anxiety, generalized anxiety disorder, panic disorder, agoraphobia and hypochondria, PTSD, and major depressive disorder. ${ }^{5,18,19-21}$ In sum, IU has gradually become understood as a fundamental element in models of the psychopathology of anxiety-related disorders. There is a growing body of evidence that IU contributes to the symptoms of various anxiety-related disorders and can be considered a transdiagnostic construct. ${ }^{7,21}$

\section{Objectives}

In the present study, we aimed to investigate the psychometric properties of the Brazilian version of the IUS-12 and provide evidence of the validity and reliability of its scores. We conducted confirmatory factor analysis (CFA) to test whether scores fit the theoretically established two-factor structure of the scale in the Brazilian setting. ${ }^{7,10}$ Internal consistency was examined using Cronbach's alpha. Additionally, the study aimed to investigate the relationships between IU and conceptually-related constructs through correlations with other related scales. We also analyzed sex and age differences in the IUS-12 scores.

Our first hypothesis was that the scale would obtain at least good internal consistency, in common with the English version of the IUS-12.11 Second, we expected that the IUS-12 for use in Brazil would replicate the two-factor structure (Prospective and Inhibitory IU subscales), also matching the original scale. Third, we also predicted that the IUS-12 would demonstrate acceptable convergent validity, indicated by at least moderate positive correlations with constructs from anxiety-related disorders, as have been reported in previous studies. ${ }^{7,21}$ Fourth, we expected women would have higher scores on the IUS-12, considering their higher prevalence rates of anxiety-related disorders. ${ }^{22}$ We also hypothesized that people could develop tolerance of uncertainty over time ${ }^{7}$ and that this would be reflected in the IUS-12 scores across different age groups. 


\section{Method}

\section{Participants and data collection}

Participants were sourced with web-based advertising through social media, and data collection was conducted via an online research platform. Access to SurveyMonkey's technology resources is only permitted through secure connectivity (e.g., virtual private network [VPN], secure shell [SSH]) and requires multifactor authentication. Data is encrypted at rest and in transit using secure transport layer security (TLS) cryptographic protocols. SurveyMonkey maintains and regularly reviews and updates its information security policies, on at least an annual basis.

Scholars from various parts of Brazil were invited to collaborate with the research by email and to share the invitation containing the weblink with their students and colleagues. Mean age was 26.74 years (standard deviation $[S D]=8.36$, median $=24$ ) and participants did not receive any gratification for their participation.

The research link was accessed by 1022 people, but only 712 of them completed all of the instruments (69\%). Inclusion criteria were adults (18-59 years old) with internet access. In view of this, eight participants $\geq 60$ years old $(1.12 \%)$ were excluded from the sample because they were defined as elderly. The resulting sample ( $n=704$ ) primarily self-identified as female $(80.11 \%)$ and equated to approximately 58 participants per item, which is in accordance with guidelines for performance of factor analyses. ${ }^{23}$

The study was approved by the ethics committee at the Universidade Federal do Rio Grande do Sul (UFRGS; protocol 2.122.254) and complies with national legislation. Informed consent was provided online before the beginning of the questionnaires. Participants completed the IUS-12 (Short Version in Brazilian Portuguese), a sociodemographic information questionnaire, and several self-report measures of related constructs which were presented at random.

\section{Measures}

Intolerance of Uncertainty Scale, Short Version (IUS-12)

The Brazilian Portuguese version of the IUS-12 was administered in order to collect evidence of its validity and reliability. The cross-cultural adaptation process of the IUS-12 began after consent was granted in writing by one of the original authors of the English version of the IUS-12. The cross-cultural adaptation process was divided into 7 steps which were intended to ensure semantic equivalency with the original IUS-12 in English (unpublished observations). ${ }^{24}$ The translation process was refined by careful analysis of cultural aspects that could influence the Brazilian population's understanding of the items. The scale appears to have excellent content validity, according to evaluations carried out by independent translators, cognitive-behavioral psychotherapists, and experts in psychological evaluation. Additionally, the IUS-12 received excellent reviews in a target population assessment, which also demonstrated that the scale has excellent face validity.

\section{The Obsessive-Compulsive Inventory-Revised Version (OCI-R)}

This scale measures OCD-related symptoms and has been translated into Brazilian Portuguese and validated by Souza et al. ${ }^{25}$ Its 18 items are rated on a five-point Likert scale on which participants choose the extent to which the symptoms have bothered them in the past month, ranging from zero (not at all) to four (extremely). This inventory had a six-factor structure revealed by exploratory factor analysis of data from a clinical and non-clinical sample and it obtained good test-retest reliability $(a=0.70)$. Data on its psychometric properties suggest that the Brazilian version of OCI-R can be used as a screening tool for OCD that discriminates between patients who have been diagnosed with OCD and patients with other anxiety disorders and healthy individuals.

\section{Obsessional Beliefs Questionnaire (OBQ-44)}

This is a 44-item self-report questionnaire divided into three subscales: a) responsibility and threat estimation, b) importance and control of thoughts, and c) perfectionism/certainty. The items are graded using a seven-point Likert scale ranging from one (totally disagree) to seven (totally agree). The OBQ44 demonstrated excellent internal consistency: for the general scale ( $a=0.95)$, for the responsibility and threat estimation subscale ( $a=0.91)$, for the importance and control of thoughts subscale ( $a=0.91$ ), and for the perfectionism/certainty subscale $(a=0.90) .{ }^{26}$

\section{Generalized Anxiety Disorder Questionnaire (GAD-7)}

This questionnaire was translated and validated for Brazil by Moreno et al. ${ }^{27}$ Its seven items investigate GAD symptoms in the previous week and are graded on a four-point Likert scale ranging from zero (rarely) to three (almost every day). Confirmatory factor analysis indicated a one-dimensional model, as was the case with the original scale. The questionnaire showed good reliability $(a=0.916){ }^{27}$

\section{Penn State Worry Questionnaire (PSWQ)}

The PSWQ is used for assessment of worry and has been translated and validated for Brazil by Castillo et al. ${ }^{28}$ It is a 16 -item measure ranked on a five-point 
Likert scale and it has good internal consistency (a $=0.84)$. Confirmatory factor analysis of the Brazilian version revealed a three-factor solution, but in view of the good correlations between the factors and the scale total score, associated with moderate correlations between the total score and another anxiety measure (State-Trait Anxiety Inventory), the PSWQ is considered to refer to a single distinctive construct. ${ }^{18}$

\section{Social Phobia Inventory (SPIN)}

This instrument was translated and adapted for Brazil by Osório et al. ${ }^{29}$ Its 17 items are measured on a fivepoint Likert scale, ranging from zero to four. The SPIN is used to evaluate fear, avoidance, and physiological symptoms associated with social anxiety disorder. The instrument demonstrated excellent internal consistency for the general scale $(a=0.90)$, and good levels for the fear subscale $(a=0.80)$, for the avoidance subscale ( $a$ $=0.78)$, and for the physiological symptoms subscale $(a=0.71) .^{29}$

\section{Data analysis}

Preliminary statistical analyses were performed with the Statistical Package for the Social Sciences (SPSS), version 22.0 for Windows. The IUS-12 did not exhibit robust deviations from normality (skew $\mathrm{z}$ score $=1.86$ ) and so means, SDs, and corrected itemtotal correlations were computed for all items of the IUS-12. Frequencies of participants were calculated for each sociodemographic category, designated in accordance with Brazilian Institute of Geography and Statistics (IBGE - Instituto Brasileiro de Geografia e Estatística) methods: gender, age range, race/skin color, educational level, and marital status. Gender differences in scores were analyzed for all subscales using the independent $t$ test. Effect sizes were calculated (Cohen's d) as indicators of the magnitude of differences between gender groups and Levene's test was used to assess equality of variances. Analyses of variance (ANOVA) were conducted using the Tukey test adjusted for multiple comparisons between groups to examine age differences with the independent variable stratified into five groups (Group 1: ages 18 to 24; Group 2: 25 to 29; Group 3: 30 to 39; Group 4: 40 to 49; and Group 5: 50 to 59). Additionally, Cronbach's Alpha was calculated for the IUS-12 and its subscales to determine internal consistency. Spearman correlations were calculated to obtain indices for relationships between the variables and evidence of convergent validity, considering the asymmetry of some of the measures used (OCI-R, SPIN, and PSWQ). Additionally, a hierarchical regression analysis was conducted and all variance inflation factors (VIF) were calculated to further investigate IU and its relationship with gender, age, and related constructs. Furthermore, CFA was conducted using R Software (R Core Team), ${ }^{30}$ in order to evaluate the dimensions of the IUS-12. The maximum likelihood estimation (MLR) method was employed to evaluate the model fit, and the following fit indices were calculated: the chi-square to degrees of freedom ratio $\left(\mathrm{X}^{2} / \mathrm{df}\right)$, the comparative fit index (CFI), the Tucker-Lewis index (TLI), the root mean square error of approximation (RMSEA), and the standardized root mean square residual (SRMR). The cut-offs adopted for the model fit indicators were as follows: $x^{2} / d f \leq 3, C F I / T L I \geq 0.95$ (excellent fit) or 0.90 (good fit); SRMR $<0.10$, and RMSEA $<0.08$. The minimum factor loading accepted was 0.30. All statistical analyses were considered significant at a 0.05 level.

\section{Results}

\section{IUS-12 descriptive statistics}

The participants' mean score for the IUS-12 was 38.70 (95\% confidence interval [95\%CI] 37.95-39.46, $\mathrm{SD}=10.20$, median $=39$, with skewness of -0.172 (standard error $[\mathrm{SE}]=0.092$ ) and kurtosis of -0.628 (SE $=0.184$ ). In order to investigate gender differences, we carried out independent sample $t$ tests with the Alpha error set at 0.05. Females had a higher mean score on the IUS-12 (mean $[M]=39.44 ; \mathrm{SD}=9.98$ ) than males $(M=35.75 ; S D=10.57)$. The difference is statistically significant, but the effect size is small: $t_{(702)}=3.87$; $\mathrm{p}<0.001, \mathrm{~d}=0.36,95 \% \mathrm{CI} 0.18-0.55$. Females also scored higher on the Inhibitory IU subscale $(M=15.05$; $\mathrm{SD}=5.37)$ than males $(M=12.87, \mathrm{SD}=5.30): \mathrm{t}_{(702)}$ $=4.30 ; \mathrm{p}<0.001, \mathrm{~d}=0.40,95 \% \mathrm{CI} 0.22-0.59$. On the Prospective IU subscale, females once more had higher scores $(M=24.38, S D=5.53)$ than males $(M=22.87$, $\mathrm{SD}=6.13): \mathrm{t}_{(198.83)}=2.66 ; \mathrm{p}=0.008, \mathrm{~d}=0.2695 \% \mathrm{CI}$ $0.08-0.45$. For this comparison, Levene's test indicated unequal variances $(F=6.09, p=0.014)$ so degrees of freedom were adjusted from 702 to 198.83 .

With regard to the other scales administered, females scored significantly $(p<0.05)$ higher on the OCI-R Obsessing subscale $\mathrm{t}_{(702)}=2.12$, the OBQ-44 (total score) $\mathrm{t}_{(702)}=2.23$, the OBQ-44 Importance and control of thoughts subscale $t_{(702)}=2.39$, the OBQ44 Perfectionism/Certainty subscale $t_{(702)}=2.61$, the GAD-7 $\mathrm{t}_{(702)}=3.85$, the SPIN $\mathrm{t}_{(702)}=3.34$, and the PSWQ $\mathrm{t}_{(702)}=5.88$.

Demographic data on race/skin color were collected using the categories employed by IBGE, as follows: white $(66.5 \%)$, black $(7.1 \%)$, yellow (Asians) 
(2\%), pardo (which refers to mixed-race Brazilians, commonly with varying proportions of white, black, and/or indigenous Brazilian ancestry) (24\%), and indigenous (which is a racial category, rather than a skin color) (3\%). The mean age of participants was 26.74 $(\mathrm{SD}=8.36)$ years and most were women $(80.1 \%)$. Additionally, $34.2 \%$ had started but not completed a university degree, $16.8 \%$ had graduated from high school, $16.2 \%$ had a university diploma, $19.9 \%$ had a graduate diploma, $9.4 \%$ had started but not completed a graduate degree, and $0.4 \%$ had only completed elementary education. Data were collected in 25 of the 26 Brazilian states and the Federal District (Distrito Federal). Participants were mostly from the south and southeast regions, with greatest participation from Rio Grande do Sul (23.9\%), São Paulo (21.9\%), and Minas Gerais $(13.5 \%)$ states. There were no participants from the state of Roraima.

\section{Age effects}

One-way ANOVA between groups was used to test for the presence of significant differences in IUS-12 scores between the range of ages present in the study sample. Since most studies are conducted using undergraduate samples, this analysis was conducted to investigate possible differences in uncertainty management across different periods in life. With this in mind, five age groups were created (Group 1: 18 to 24; Group 2: 25 to 29; Group 3: 30 to 39; Group 4: 40 to 49; and Group 5: 50 to 59$)$. There was a statistically significant difference $F_{(4)}$ $=7.85, \mathrm{p}<0.001$ in IUS-12 scores across the groups. The assumption of homogeneity was met for the IUS12 and subscales $(p>0.05)$. Post hoc comparisons using Tukey's honestly significant difference (Tukey's HSD) test indicated that the mean score for Group 5 participants $(M=29.61$; $S D=11.07)$ was significantly different ( $p \leq 0.001$ ) from the mean score for Group $1(M=40 ; S D=9.45 ; d=1.09)$ and Group $2(M=$ 36.48; SD $=10.58, d=.82)$. The same pattern emerged in comparisons between the two subscales. On the IU Prospective Subscale, Group $5(M=19.83$; $S D=6.8)$ obtained significantly lower $(p<.05)$ scores than Group 1 $(M=24.64 ; S D=5.30 ; d=0.89)$. For the IU Inhibitory subscale, Group 5 scores $(M=9.77 ; S D=5.83)$ were also significantly lower $(p<0.001)$ than Group 1 ( $M=$ $15.36 ;$ SD $=5.20 ; d=1.06)$.

\section{Evidence regarding the internal structure Internal consistency}

Cronbach's alpha was 0.88 for all 12 items of the scale, 0.79 for the Prospective IU subscale, and 0.86 for the Inhibitory IU subscale. None of the items had a corrected item-total correlation (CITC) lower than 0.40 .

\section{IUS-12 factor structure}

Confirmatory factor analysis was conducted using the lavaan package ${ }^{31}$ in $\mathrm{R}$ Software, ${ }^{30}$ to probe the factor structure of the IUS-12. The predicted two-factor structure of the scale is based on the theory of IU and extensive evidence of this factor solution for the IUS12.7,10 Therefore, a two-dimensional model was tested to confirm the well-established Prospective IU (items 1, 2, 4, 5, 8, 9 and 11) and Inhibitory IU (items 3, $6,7,10,12)$ factors. ${ }^{21}$ Factor reliability was calculated using Cronbach's alpha (the minimum for satisfactory reliability is 0.70$){ }^{32}$

The results suggested a good model fit, indicating that the factor structure could be conceptualized as a two-dimensional model containing Prospective and Inhibitory IU. Model fit: CFI $=0.947$, TLI $=0.931$, RMSEA $=0.069,90 \%$ CI RMSEA $=[0.60,0.079]$; SRMR $=0.043$. Factor loadings varied from 0.46 to 0.78 , and the correlation between factors was 0.83 . Factor loadings and standard errors are presented in Table 1.

\section{Evidence regarding relationships with conceptually-related constructs}

Evidence of convergent validity was examined via relationships with related variables. Spearman correlation coefficients suggested strong relationships between the IUS-12 and measures of anxiety-related symptoms (Table 2). Evidence for convergent validity of the IUS-12 has been previously examined in Canada, ${ }^{18}$ the US, ${ }^{33}$ and Australia ${ }^{21}$ through correlations of the scale with anxiety-related constructs.

Table 1 - Standardized factor loadings and standard errors for the 12 items of the Intolerance of Uncertainty Scale, per subscale

\begin{tabular}{ccc}
\hline & \multicolumn{2}{c}{ Subscale } \\
\cline { 2 - 3 } Item & Prospective IU & Inhibitory IU \\
\hline 1 & $0.59(1.0)$ & \\
2 & $0.46(0.071)$ & \\
4 & $0.60(0.098)$ & \\
5 & $0.65(0.102)$ & \\
8 & $0.61(0.105)$ & \\
9 & $0.62(0.088)$ & \\
11 & $0.54(0.097)$ & $0.73(1.0)$ \\
3 & & $0.76(0.056)$ \\
6 & & $0.78(0.053)$ \\
7 & & $0.75(0.052)$ \\
10 & & $0.70(0.052)$ \\
12 & & \\
\hline
\end{tabular}

Prospective IU = Intolerance of Uncertainty, Prospective Subscale; Inhibitory IU = Intolerance of Uncertainty, Inhibitory Subscale. $p<0.001$. 
The analysis demonstrated strong correlations with measures of worry (PSWQ), anxiety (GAD-7), obsessions and compulsions (OBQ-44, OCI-R), and social phobia (SPIN). Specifically, the IUS-12 correlated positively with the Perfectionism/Certainty subscale of the OBQ$44(\rho=0.65, p<0.01)$, which measures dysfunctional beliefs about uncertainty, perfectionism, and need for control. ${ }^{27}$ Similarly, the Inhibitory IU subscale, which is related to uncertainty paralysis and avoidance demonstrated strong correlation with the Social Phobia Inventory ( $\rho=0.58, p<0.01$ ), and the Prospective IU subscale, which is related to cognitive appraisals of future uncertainty, was also strongly correlated with the PSWQ $(\rho=0.66, p<0.01)$.

\section{Hierarchical regression analysis}

Relationships between the scores of the IUS-12 and scores of related variables were examined using hierarchical multiple regression analysis. Age and sex were entered as independent variables in the first step, and all other related constructs were entered in the second step. Outcome variables were the Prospective IU and Inhibitory IU subscales.
The results (Table 3 ) indicated that the step one predictors only explained approximately $3.8 \%$ of the variance in Prospective IU scores $\left(\Delta R^{2}=0.038, F_{(2)}\right.$ $=14.31, p<0.001)$. When the other variables were introduced, the model explained an additional $54.2 \%$ of Prospective IU variance $\left(\Delta \mathrm{R}^{2}=0.54, \mathrm{~F}_{(15)}=53.84\right.$, $p<0.001)$. Age and sex explained $6.8 \%$ of Inhibitory IU variance $\left(\Delta R^{2}=0.068, F_{(2)}=25.58, p<0.001\right)$. Addition of the related constructs to the regression model explained an additional $51 \%$ of Inhibitory IU variance $\left(\Delta R^{2}=0.51, F_{(15)}=48.87, p<0.001\right)$.

The PSWQ (Positive Items subscale) score was the most critical predictor of Prospective IU and uniquely explained $11.56 \%$ of variance. It was also found that the OBQ-44 Perfectionism/Certainty subscale significantly predicted Prospective IU scores, independently of all other independent variables $(\beta=0.027 ; p<0.001)$. A robust relationship was also found between PSWQ (Positive Items subscale) scores and the Inhibitory IU scores $(\beta=0.23 ; p<0.001)$ and between OBQ-44 Perfectionism/Certainty scores and Inhibitory IU scores, explaining $5 \%$ of the variance. There were no indications of problems with multicollinearity (all VIF < 10).

Table 2 - Spearman correlations $(\rho)$ between IUS-12 and related constructs $(n=704)$

\begin{tabular}{|c|c|c|c|}
\hline & $\begin{array}{l}\text { IUS-12 } \\
\text { (Total) }\end{array}$ & $\begin{array}{c}\text { Prospective } \\
\text { IU } \\
\end{array}$ & $\begin{array}{c}\text { Inhibitory } \\
\text { IU }\end{array}$ \\
\hline 1. IUS-12 (total) & - & 0.91 & 0.91 \\
\hline 2. Prospective IU & - & - & 0.67 \\
\hline 3. Inhibitory IU & - & - & - \\
\hline 4. OCI-R & 0.58 & 0.52 & 0.54 \\
\hline 5. OCI-R Checking & 0.38 & 0.33 & 0.36 \\
\hline 6. OCI-R Hoarding & 0.43 & 0.37 & 0.42 \\
\hline 7. OCI-R Washing & 0.23 & 0.19 & 0.23 \\
\hline 8. OCI-R Ordering & 0.33 & 0.34 & 0.27 \\
\hline 9. OCIR-Neutralizing & 0.27 & 0.23 & 0.26 \\
\hline 10. OCI-R Obsessing & 0.55 & 0.48 & 0.53 \\
\hline 11. OBQ-44 & 0.65 & 0.58 & 0.62 \\
\hline 12. OBQ-44 ICT & 0.53 & 0.43 & 0.54 \\
\hline 13. OBQ-44 RT & 0.58 & 0.53 & 0.53 \\
\hline 14. OBQ-44 PC & 0.63 & 0.59 & 0.57 \\
\hline 15. GAD-7 & 0.58 & 0.54 & 0.53 \\
\hline 16. SPIN & 0.58 & 0.49 & 0.58 \\
\hline 17. PSWQ (Total) & 0.68 & 0.66 & 0.59 \\
\hline 18. PSWQ Negative Items & -0.45 & -0.47 & -0.35 \\
\hline 19. PSWQ Positive Items & 0.69 & 0.65 & 0.60 \\
\hline
\end{tabular}

GAD-7 = Generalized Anxiety Disorder Questionnaire; Inhibitory IU = IUS-12 Inhibitory subscale; IUS-12 = Intolerance of Uncertainty Scale Short Version; OBQ-44 = Obsessional Beliefs Questionnaire; OBQ-44 ICT = OBQ-44 Importance and Control of Thoughts Subscale; OBQ-44 RT = OBQ-44 Responsibility and Threat Estimation subscale; OBQ-44 PC = OBQ-44 Perfectionism/Certainty subscale; OCI-R = Obsessive-Compulsive Inventory Revised; Prospective IU = IUS12 Prospective subscale; PSWQ = Penn State Worry Questionnaire; PSWQ Negative Items = reflects absence of worry; PSWQ Positive Items = reflects presence of worry; SPIN = Social Phobia Inventory.

All correlations were significant at the 0.01 level (2-tailed). 
Psychometric properties of the IUS-12 for Brazil - Kretzmann et al.

Table 3 - Summary of hierarchical regression analysis for variables predicting Inhibitory IU and Prospective IU from age, sex, and all other subscales

\begin{tabular}{|c|c|c|c|c|c|c|c|c|}
\hline \multirow[b]{2}{*}{ Variables } & \multirow[b]{2}{*}{$\Delta \mathbf{R}^{2}$} & \multicolumn{3}{|c|}{ Prospective IU } & \multirow[b]{2}{*}{$\Delta \mathbf{R}^{2}$} & \multicolumn{2}{|c|}{ Inhibitory IU } & \multirow[b]{2}{*}{$95 \% \mathrm{CI}$} \\
\hline & & $\boldsymbol{\beta}$ & $\mathbf{p}$ & $95 \% \mathrm{CI}$ & & $\boldsymbol{\beta}$ & $\mathbf{p}$ & \\
\hline Step 1 & 0.038 & & & & 0.06 & & & \\
\hline Age & & -0.18 & $<0.001$ & -0.17 to -0.07 & & -0.21 & $<0.001$ & -0.18 to -0.09 \\
\hline Sex & & -0.09 & 0.01 & -2.3 to -0.20 & & -0.15 & $<0.001$ & -3.04 to -1.0 \\
\hline Step 2 & 0.54 & & & & 0.51 & & & \\
\hline Age & & -0.02 & 0.48 & -0.05 to 0.02 & & -0.014 & 0.62 & -0.04 to 0.02 \\
\hline Sex & & 0.05 & 0.06 & -0.04 to 1.4 & & -0.03 & 0.23 & -1.1 to 0.28 \\
\hline OCI-R Checking & & -0.01 & 0.61 & -0.16 to 0.09 & & 0.03 & 0.25 & -0.5 to 0.20 \\
\hline OCI-R Hoarding & & -0.001 & 0.97 & -0.12 to 0.11 & & 0.04 & 0.16 & -0.03 to 0.19 \\
\hline OCI-R Washing & & 0.002 & 0.95 & -0.18 to 0.19 & & 0.01 & 0.67 & -0.14 to 0.22 \\
\hline OCI-R Ordering & & 0.06 & 0.03 & 0.008 to 0.24 & & -0.03 & 0.26 & -0.17 to 0.04 \\
\hline OCI-R Neutralizing & & 0.017 & 0.57 & -0.12 to 0.22 & & 0.00 & 0.99 & -0.17 to 0.17 \\
\hline OCI-R Obsessing & & 0.006 & 0.86 & -0.1 to 0.12 & & 0.03 & 0.42 & -0.06 to 0.15 \\
\hline OBQ-44 ICT & & -0.06 & 0.12 & -0.05 to 0.006 & & 0.14 & 0.001 & 0.02 to 0.07 \\
\hline OBQ-44 RT & & 0.033 & 0.49 & -0.17 to 0.035 & & -0.04 & 0.41 & -0.03 to 0.01 \\
\hline OBQ-44 PC & & 0.27 & $<0.001$ & 0.05 to 0.09 & & 0.22 & $<0.001$ & 0.035 to 0.07 \\
\hline GAD-7 & & 0.07 & 0.07 & -0.007 to 0.14 & & 0.07 & 0.08 & -0.01 to 0.13 \\
\hline SPIN & & 0.10 & 0.002 & 0.01 to 0.06 & & 0.21 & $<0.001$ & 0.05 to 0.10 \\
\hline PSWQ (Positive Items) & & 0.34 & $<0.001$ & 0.14 to 0.24 & & 0.23 & $<0.001$ & 0.07 to 0.17 \\
\hline PSWQ (Negative Items) & & -0.12 & $<0.001$ & -0.27 to -0.09 & & 0.004 & 0.89 & -0.08 to 0.09 \\
\hline
\end{tabular}

$95 \% \mathrm{CI}=95 \%$ confidence interval; GAD-7 = Generalized Anxiety Disorder Questionnaire; Inhibitory IU = IUS-12 Inhibitory subscale; IUS-12 = Intolerance of Uncertainty Scale Short Version; Prospective IU = IUS-12 Prospective subscale; OCI-R = Obsessive-Compulsive Inventory Revised; OBQ-44 = Obsessional Beliefs Questionnaire; OBQ-44 ICT = OBQ-44 Importance and control of thoughts subscale; OBQ-44 RT = OBQ-44 Responsibility and Threat Estimation subscale; OBQ-44 PC = OBQ-44 Perfectionism/Certainty subscale; PSWQ = Penn State Worry Questionnaire; PSWQ Negative Items = reflects absence of worry; PSWQ

Positive Items $=$ reflects presence of worry; SPIN $=$ Social Phobia Inventory.

Dependent variables: Prospective IU and Inhibitory IU.

Step 1 predictors: age and sex; Step 2 predictors: all other scales were entered along with step 1 predictors

$\mathrm{p}<0.01$.

\section{Discussion}

The present study aimed to assess the psychometric properties of the Brazilian-Portuguese version of the Brazilian Intolerance of Uncertainty Scale - Short Version. The scores from the IUS-12 were compared across five different age groups. The statistically significant differences in scores between Group 5 and Groups 1 and 2 suggest that intolerance of uncertainty may manifest itself in different ways over the course of development and that people could develop tolerance of uncertainty over time. Since most studies are conducted using undergraduate samples, research in older communities is lacking. Research is needed to investigate the trajectory of IU over the course of development, considering the neurodevelopmental differences in uncertainty management between different ages. ${ }^{7}$ Future studies could evaluate whether elements such as stress might influence the construct's temporal stability, along with the presence of emotional disorders. Correspondingly, future studies should investigate how people manage uncertainty throughout their lifespan and explore techniques for development of tolerance of uncertainty.
Concerning evidence on the internal structure of the IUS-12, the CFA results supported the two-dimensional model previously reported for the original scale. ${ }^{10}$ The results demonstrated a two-factor structure comprising Prospective IU, which evaluates desire for predictably, and Inhibitory IU, which assesses uncertainty paralysis.

Further psychometric evaluation of the scale suggested that it possesses high internal consistency and good convergent validity, as indicated by large positive correlations with related constructs. As hypothesized, the study found strong correlations with measures of worry (PSWQ), anxiety (GAD-7), obsessions and compulsions (OBQ-44, OCI-R), and social phobia (SPIN), indicating convergent evidence based on relationships with other variables. These findings are consistent with theoretical assumptions in the literature, demonstrating that people with high levels of IU usually report similar levels of worry and other anxiety-related constructs. ${ }^{7}$

It has been suggested that each of the IUS12 subscales would be correlated with specific pathologies. ${ }^{34}$ However, contrary to this expectation, Inhibitory IU and Prospective IU were equally correlated with the GAD-7 questionnaire, although Prospective IU had a stronger correlation with the 
PSWQ than Inhibitory IU. The GAD-7 is based on Diagnostic and Statistical Manual of Mental Disorders criteria and encompasses both cognitive and behavioral inhibition anxiety-related aspects, which could explain the equivalency of the correlation scores of the two IU subscales. Moreover, research suggests that people with higher levels of IU have a predisposition to engage in cycles of worry, which may lead to maintenance and development of GAD. ${ }^{35}$ Correspondingly, Inhibitory IU was more strongly correlated with the Social Phobia Inventory when compared to Prospective IU, which is in accordance with evidence that Inhibitory IU is more associated with symptoms of social anxiety. ${ }^{36}$

Moreover, Prospective IU had a similar but slightly weaker relationship with the OCI-R Checking subscale than Inhibitory IU. These results coincide with Fourtounas and Thomas' ${ }^{\prime 14}$ investigation of the relationship between the IUS-12 subscales and checking behaviors through regression analysis, which demonstrated that both subscales explained similar unique variance in checking behaviors. Thus, results suggest that both Inhibitory IU and Prospective IU are related to neutralization strategies, comprising behavioral and cognitive aspects of uncertainty management.

These outcomes also contribute to the transdiagnostic understanding of IU through its association with various anxiety-related constructs. Therefore, the current study provides evidence of the relationship between intolerance of uncertainty and measures of anxiety symptoms in South America that is comparable with previous results acquired with Canadian and Australian samples. ${ }^{7,21}$ Thus, future research is needed to examine the stability of IU across different cultures, also aiming to investigate the equivalency of results in developed and developing countries. A standardized measure of IU should contribute to a cross-cultural comparison with evidence of factor stability and equivalency of scores across countries.

The adaptation of the IUS-12 for use in Brazil has clinical implications concerning intolerance of uncertainty and cognitive-behavioral treatments that focus on anxiety-related disorders. Trait IU, and consequently fear of the unknown, as measured by the IUS-12 can be conjointly addressed in clinical interventions focusing on IU-related fears. ${ }^{21,35}$ Furthermore, anxiety-related treatment protocols in Brazil could develop strategies aiming to incorporate uncertainty management and to diminish misconceptions about lack of information and its harmful consequences. ${ }^{36}$

The results of the hierarchical regression analysis supported the existence of relationships between IU, GAD, and worry as measured by the PSWQ. Mainly, worry and obsessions levels were predictors of the scores on both IU subscales, independently of other variables. Intolerance of uncertainty is postulated as a central feature in development and maintenance of GAD and the results are in accordance with previous studies that have addressed the association between IU and worry. ${ }^{11,34,37}$

The current investigation has limitations. On the one hand, despite extensive effort to guarantee a representative sample, the study had a predominance $(80 \%)$ of female participants. This, however, appears to be a common occurrence in scientific studies of IU. 10,11,38 On the other hand, participants were given access to the research link online, which was intended to ensure diversity of the sample, extending it beyond university students. Moreover, future studies should use clinical samples to evaluate associations between IU and anxiety-related constructs further, aiming to investigate the transdiagnostic nature of IU in greater depth. ${ }^{35}$ Correspondingly, longitudinal research is necessary to investigate possible changes in IU over time.

Another limitation is that supplementary anxietyrelated constructs were not included in the study (e.g., anxiety sensitivity, rumination, fear of negative evaluation). Future studies in Brazil should aim to include measures of depression and personality (e.g., neuroticism). This would be consistent with studies showing that symptoms of worry social anxiety, worry, obsessive-compulsive disorder, and depression may have relationships with neuroticism mediated by IU. ${ }^{34}$

Additionally, the study relied solely on online selfreport measures and did not incorporate behavioral observations. It has been suggested that high levels of IU may be associated with low performance in behavioral tasks and difficulty in decision-making processes. ${ }^{15}$ Future research should aim to develop experimental studies that investigate IU in behavioral tasks along with related biological markers such as heart rate and electrical skin conductance. ${ }^{36}$ Moreover, studies have associated IU with psychophysiological measures, indicating possible changes in reward processing. ${ }^{5}$ Investigating psychophysiological measures in relation to IU could help to elucidate its relationship with central mechanisms of anxietyrelated psychopathology.

Despite these limitations, the results provide evidence of the reliability and validity of the IUS-12 for use in Brazil. The IUS-12 demonstrated excellent internal consistency, good construct validity, and good convergent validity through investigation of its psychometric properties. This instrument is the first in Brazil that offers the possibility of measuring trait IU and has several implications for both clinical practice and research. 


\section{Acknowledgements}

The research presented in this manuscript was partially funded by the Conselho Nacional de Desenvolvimento Científico e Tecnológico (CNPq) and the Coordenação de Aperfeiçoamento de Pessoal de Nível Superior (CAPES) public research funding bodies. Roberta Pozzi Kretzmann declares receipt of grants from CNPq during the course of the study.

\section{Disclosure}

No conflicts of interest declared concerning the publication of this article.

\section{References}

1. Yoe C. Primer on risk analysis: decision making under uncertainty. Boca Raton: CRC Press: 2012.

2. Tonetto LM, Kalil LL, Melo WV, Schneider DDG, Stein LM. O papel das heurísticas no julgamento e na tomada de decisão sob incerteza. Estud Psicol (Campinas). 2006;23:181-9.

3. Mushtaq F, Bland AR, Schaefer A. Uncertainty and cognitive control. Front Psychol. 2011;2:249.

4. Carleton RN. Into the unknown: A review and synthesis of contemporary models involving uncertainty. J Anxiety Disord. 2016:39:30-43.

5. Tanovic E, Gee DG, Joormann J. Intolerance of uncertainty: neural and psychophysiological correlates of the perception of uncertainty as threatening. Clin Psychol Rev. 2018;60:87-99.

6. Freeston $\mathrm{MH}$, Rhéaume L, Letarte $\mathrm{H}$, Dugas MJ, Ladouceur R. Why do people worry? Pers Individ Dif. 1994;17:791-802.

7. Shihata S, McEvoy PM, Mullan BA, Carleton RN. Intolerance of uncertainty in emotional disorders: what uncertainties remain?. J Anxiety Disord. 2016;41:115-24.

8. Carleton RN. Fear of the unknown: One fear to rule them all? J Anxiety Disord. 2016;41:5-21.

9. Clark DA, Beck AT. Cognitive therapy of anxiety disorders. New York: Guildford Press; 2010.

10. Carleton RN, Norton MAPJ, Asmundson GJG. Fearing the unknown: A short version of the Intolerance of Uncertainty Scale. J Anxiety Disord. 2007:21:105-17.

11. McEvoy PM, Mahoney AEJ. To be sure, to be sure: intolerance of uncertainty mediates symptoms of various anxiety disorders and depression. Behav Ther. 2012;43:533-45.

12. Birrell J, Meares K, Wilkinson A, Freeston M. Toward a definition of intolerance of uncertainty: a review of factor analytical studies of the Intolerance of Uncertainty Scale. Clin Psychol Rev. 2011;31:1198-208.

13. Khawaja NG, Yu LNH. A comparison of the 27-item and 12-item intolerance of uncertainty scales. Clin Psychol (Aust Psychol Soc). 2010;14:97-106.

14. Fourtounas A, Thomas SJ. Cognitive factors predicting checking, procrastination and other maladaptive behaviours: prospective versus inhibitory intolerance of uncertainty. J Obsessive Compuls Relat Disord. 2016;9:30-5.

15. Thibodeau MA, Carleton RN, Gómez-Pérez L, Asmundson GJG. "What if I make a mistake?": intolerance of uncertainty is associated with poor behavioral performance. J Nerv Ment Dis. 2013;201:760-6.

16. Sexton KA, Dugas MJ. Defining distinct negative beliefs about uncertainty: validating the factor structure of the intolerance of uncertainty scale. Psychol Assess. 2009;21:176-86.

17. Thibodeau MA, Carleton RN, McEvoy PM, Zvolensky MJ, Brandt $\mathrm{CP}$, Boelen PA, et al. Developing scales measuring disorderspecific intolerance of uncertainty (DSIU): a new perspective on transdiagnostic. J Anxiety Disord. 2015;31:49-57.

18. Fetzner MG, Horswill SC, Boelen PA, Carleton RN. Intolerance of uncertainty and PTSD symptoms: exploring the construct relationship in a community sample with a heterogeneous trauma history. Cognit Ther Res. 2013;37:725-34.

19. Counsell $A$, Furtado $M$, Iorio $C$, Anand $L$, Canzonieri A, Fine $A$ et al. Intolerance of uncertainty, social anxiety, and generalized anxiety: differences by diagnosis and symptoms. Psychiatry Res. 2017;252:63-9.

20. Pepperdine $\mathrm{E}$, Lomax C, Freeston $\mathrm{MH}$. Disentangling intolerance of uncertainty and threat appraisal in everyday situations. J Anxiety Disord. 2018;57:31-8

21. Mahoney AE, McEvoy PM. Trait versus situation-specific intolerance of uncertainty in a clinical sample with anxiety and depressive disorders. Cogn Behav Ther. 2012;41:26-39.

22. American Psychiatric Association. DSM-5: Manual diagnóstico e estatístico de transtornos mentais. $5^{a}$ ed. Porto Alegre: Artmed; 2014.

23. Costello AB, Osborne JW. Best practices in exploratory factor analysis: four recommendations for getting the most from your analysis. Pract Assess Res Evaluat. 2005;10:1-9.

24. Kretzmann RP. Intolerance of Uncertainty Scale (IUS-12) para uso no Brasil: adaptação transcultural e propriedades psicométricas [dissertation]. Porto Alegre: Universidade Federal do Rio Grande do Sul; 2018.

25. Souza FP, Foa EB, Meyer E, Niederauer KG, Cordioli AV. Psychometric properties of the Brazilian Portuguese version of the Obsessive-Compulsive Inventory - Revised (OCI-R). Braz J Psychiatry. 2011;33:137-43.

26. Bortoncello CF, Braga DT, Gomes JB, Souza FP, Cordiol AV. Psychometric properties of the Brazilian version of the Obsessive Beliefs Questionnaire (OBQ-44). J Anxiety Disord. 2012:26:430-4.

27. Moreno AL, De Sousa DA, Pereira de Souza AMFL, Manfro GG, Salum GA, Koller SH, et al. Factor structure, reliability and item parameters of the Brazilian-Portuguese version of the GAD-7 questionnaire. Trends Psychol. 2016;24:367-76.

28. Castillo C, Macrini L, Cheniaux E, Landeira-Fernandez J. Psychometric properties and latent structure of the Portuguese version of the Penn Worry State Questionnaire. Span J Psychol. 2010;13:431-43.

29. Osório FL, Crippa JAS, Loureiro SR. Cross-cultural validation of the Brazilian Portuguese version of the Social Phobia Inventory (SPIN): study of the items and internal consistency. Braz J Psychiatry. 2009;31:25-9.

30. R Core Team. R: A language and environment for statistical computing. Vienna: R Foundation for Statistical Computing; 2014 [accessed 2018 Sep 26]. http://www.R-project.org/

31. Rosseel Y. Lavaan: An R package for structural equation modeling. J Stat Soft. 2012;48:1-36.

32. Hair JF, Anderson RE, Tatham RL, Black WC. Multivariate data analysis. Essex: Pearson; 2014

33. Bardeen JR, Fergus TA, Wu KD. The interactive effect of worry and intolerance of uncertainty on posttraumatic stress symptoms. Cognit Ther Res. 2013;37:742-51.

34. McEvoy PM, Erceg-Hurn DM. The search for universal transdiagnostic and trans-therapy change processes: Evidence for intolerance of uncertainty. J Anxiety Disord. 2016;41:96-107.

35. Talkovsky AM, Norton PJ. Intolerance of uncertainty and transdiagnostic group cognitive behavioral therapy for anxiety. J Anxiety Disord. 2016;41:108-14.

36. Carleton RN. The intolerance of uncertainty construct in the context of anxiety disorders: theoretical and practical perspectives. Expert Rev Neurother. 2012;12:937-47.

37. Bomyea J, Ramsawh $\mathrm{H}$, Ball TM, Taylor CT, Paulus MP, Lang AJ, et al. Intolerance of uncertainty as a mediator of reductions in worry in a cognitive behavioral treatment program for generalized anxiety disorder. J Anxiety Disord. 2015;33:90-4.

38. Gosselin $P$, Ladouceur $R$, Evers $A$, Laverdière $A$, Routhier $S$, Tremblay-Picard M. Evaluation of intolerance of uncertainty: development and validation of a new self-report measure. Anxiety Disord. 2008;22:1427-39.

\section{Correspondence:}

Roberta P. Kretzmann

Departamento de Psicologia do Desenvolvimento e da Personalidade

Universidade do Rio Grande do Sul (UFRGS)

Rua Ramiro Barcelos, 2600, sala 121

90035-003 - Porto Alegre, RS - Brazil

Tel./Fax: +55 5133085341

E-mail: robertakretzmann@gmail.com 